\title{
Múltiplos dentes impactados em paciente não sindrômico
}

\author{
Multiple impacted teeth in patient nonsyndromic \\ Múltiples dientes impactados en pacientes no sindrómicos
}

Recebido: 31/12/2020 | Revisado: 31/12/2020 | Aceito: 21/01/2021 | Publicado: 25/01/2021

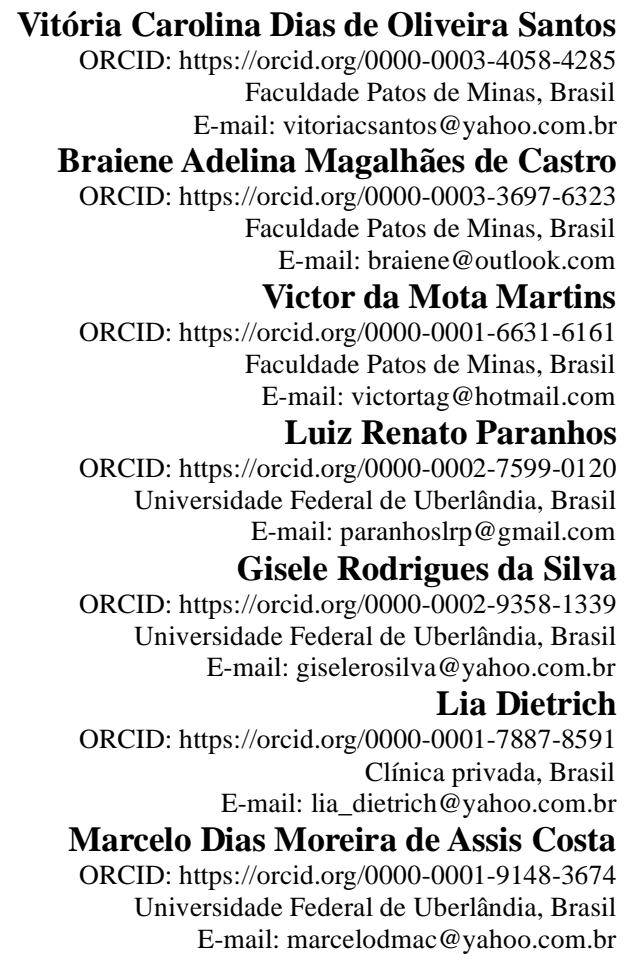

\section{Resumo}

A formação da dentição humana ocorre a partir da quinta semana de vida intrauterina, durante as fases de formação podem acontecer episódios que interferem na formação dos dentes causando alterações como a de números. Essa alteração é conhecida como dentes supranumerários, sendo caracterizada como um aumento do número de dentes, além dos normais a dentição. Eles ocasionalmente estarão impactados pela falta de espaço para irromper. $\mathrm{O}$ tratamento depende do exame clínico e complementar detalhado, sendo muitas vezes, a exodontia, a melhor opção. Existem vários exames para diagnóstico e planejamento, sendo a tomografia computadorizada a melhor opção, principalmente por oferecer uma imagem tridimensional. Nosso objetivo foi realizar uma revisão de literatura e relato de caso clínico sobre a presença de múltiplos dentes impactados em um paciente sem síndromes diagnosticada. A revisão de literatura contou com artigos na língua inglesa publicados a partir de 2006. Para o relato de caso foram utilizadas as informações do prontuário como história médica, exame clínico e tomográfico. Concluiu-se que dentes supranumerários em pacientes não sindrômicos podem estar relacionados a alterações na modulação da sinalização que controlam o desenvolvimento dental, falha dos inibidores responsáveis por apoptose dos brotos dentais, além de possível relação de herança autossômica dominante.

Palavras-chave: Dente supranumerário; Tomografia computadorizada de feixe cônico; Diagnóstico; Dente impactado.

\begin{abstract}
The formation of human dentition occurs from the fifth week of intrauterine life, during the formation phases, episodes that interfere with the formation of teeth can occur, causing changes such as numbers. This alteration is known as the supernumerary teeth, being characterized as an increase in the number of teeth, in addition to the normal teeth. They are occasionally impacted by the lack of space to break out. Treatment depends on a detailed clinical and complementary examination, and extraction is often the best option. There are several tests for diagnosis and planning, with computed tomography as the best option, mainly because it offers a three-dimensional image. Our objective was to perform a literature review and a clinical case report on the presence of multiple impacted teeth in a diagnosed patient without syndromes. The literature review included articles in the English language published since
\end{abstract}


2006. For the case report, information from medical records such as medical history, clinical examination and tomography were used. It was concluded that supernumerary teeth in non-syndromic patients may be related to changes in signaling modulation that control dental development, failure of inhibitors responsible for apoptosis of dental sprouts, in addition to a possible autosomal dominant increase.

Keywords: Supernumerary; Tooth; Cone-Beam computed tomography; Diagnosis; Tooth, impacted.

\section{Resumen}

La formación de la dentición humana se produce a partir de la quinta semana de vida intrauterina, durante las fases de formación, pueden ocurrir episodios que interfieran con la formación de los dientes, provocando cambios como los números. Esta alteración se conoce como dientes supernumerarios, caracterizándose por un aumento en el número de dientes, además de dientes normales. Ocasionalmente se verán afectados por la falta de espacio para escapar. El tratamiento depende de un examen clínico detallado y complementario, y la extracción suele ser la mejor opción. Existen varias pruebas de diagnóstico y planificación, siendo la tomografía computarizada la mejor opción, principalmente porque ofrece una imagen tridimensional. Nuestro objetivo fue realizar una revisión de la literatura y el reporte de un caso clínico sobre la presencia de múltiples dientes impactados en un paciente diagnosticado sin síndromes. La revisión de la literatura incluyó artículos en inglés publicados desde 2006. Para el reporte del caso se utilizó información de historias clínicas como historia clínica, examen clínico y tomografía. Se concluyó que los dientes supernumerarios en pacientes no sindrómicos pueden estar relacionados con cambios en la modulación de la señalización que controlan el desarrollo dentario, falla de los inhibidores responsables de la apoptosis de los brotes dentales, además de una posible relación de herencia autosómica dominante.

Palabras clave: Diente supernumerario; Tomografía computarizada de haz cónico; Diagnóstico; Diente impactado.

\section{Introdução}

O início da formação da dentição humana se dá a partir da quinta semana de vida intrauterina, onde o epitélio oral primitivo começa a se proliferar formando as bandas epiteliais e as lâminas vestibular e dentária. Posteriormente haverá fases de desenvolvimento onde ocorrem os processos de iniciação, proliferação, diferenciação, aposição e calcificação. Durante as fases de desenvolvimento alguns fatores podem interferir na formação dental, trazendo variações de tamanho, forma, cor, localização e número (supranumerários) (McCoy, 2012).

Dentes supranumerários (DS) ou hiperdontia são alterações do desenvolvimento que podem aparecer em qualquer um dos arcos dentários (Bae et al., 2017). As etiologias de formação de dentes supranumerários não são completamente definidas. As mais aceitas são a teoria de formação por meio de remanescentes da lâmina dentária que induzidos a iniciação formariam um dente extra. A teoria da hiperatividade da lâmina dentária que produziria um número anormal, no caso maior, de germes. E a teoria do resultado da divisão de um germe em desenvolvimento (Chen et al., 2019). Outra teoria os relaciona a fatores genéticos, onde estudos tem mostrado que a regulação inadequada da atividade de uma proteína pode desempenhar um papel fundamental na formação de dentes supranumerários (Al-Tamimi et al., 2017; Lu et al., 2017)

Múltiplos dentes supranumerários apresentem prevalência menor que 1\% (Açıkgöz et al., 2006). A ocorrência de múltiplos dentes supranumerários é rara e geralmente esta associada a alguma síndrome, como a síndrome de Gardner e a Displasia Cleidocraniana ou distúrbios como fissuras labiais e palatinas, porém, não exclui sua ocorrência em pacientes não sindrômicos (Bae et al., 2017; Belmehdi et al., 2018; Tanwar et al., 2017). Eles são mais encontrados no gênero masculino, na maxila anterior e região de pré-molares mandibulares (Khalaf et al., 2018; Pico et al., 2017).

Quando não relacionada a síndromes, a origem dos múltiplos dentes supranumerários pode ter uma relação hereditária autossômica dominante além das teorias da hiperatividade ou remanescentes da lâmina ("Main genetic entities associated with supernumerary teeth", 2018).

Existem consequências relacionadas a presença de múltiplos dentes supranumerários, as principais são erupção retardada, impacção dental, deslocamento dos dentes permanentes durante a erupção, reabsorção radicular e formação cística. (Açıkgöz et al., 2006; Khalaf et al., 2018; McCoy, 2012)

A impacção dental é um processo no qual o dente não consegue deslocar do seu local de desenvolvimento até seu local final no processo alveolar. Esses dentes podem ser decíduos, permanentes ou supranumerários. Ocorre com maior 
frequência na dentição permanente e o dente mais comumente relacionado é o terceiro molar (McCoy, 2012; Pico et al., 2017).

Dentre as causas de impactação temos os apinhamentos, por perda precoce de dentes decíduos, arco de Baume tipo II, prolongamento da presença do decíduo em boca e desenvolvimento maxilofacial insuficiente. Outros possíveis fatores são cistos e tumores, traumas, desordens sistêmicas e síndromes. No caso dos supranumerários sua localização atípica e o aumento do perímetro do arco necessário para sua erupção dificultam o irrompimento (Dodson, 2012; Tamimi \& ElSaid, 2009).

O tratamento depende da posição e efeito aos dentes e demais estruturas próximas. Se o dente em questão estiver interferindo na erupção, posicionamento, estética e/ou outro tratamento necessário é indicada sua extração. Em casos onde o dente supranumerário não interfere na saúde bucal e geral do paciente e o mesmo escolhe deixa-lo, devemos orienta-lo ao acompanhamento através de exames de imagem $(3,5,9)$.

A anamnese, exame clínico e exames complementares são fundamentais para o diagnóstico e planejamento cirúrgico dos dentes impactados. Os recursos mais utilizados são as radiografias intra-orais, como as periapicais ou oclusais. A técnica de Clark é útil para identificar a localização vestibular ou lingual/palatina do dente em relação aos adjacentes. Na radiografia oclusal podemos avaliar a relação horizontal do dente impactado com os demais dentes e estrutura (Boeddinghaus \& Whyte, 2008; Tamimi \& ElSaid, 2009; Tanwar et al., 2017).

A radiografia panorâmica também oferece uma imagem bidimensional, é indicada para o planejamento de procedimentos em relação aos dentes impactados pois oferece uma visão geral dos arcos, possibilitando a correlação do dente impactado com os demais dentes e estruturas próximas, mas não permite a localização precisa dos dentes (Boeddinghaus \& Whyte, 2008; Meara, 2012).

A tomografia computadorizada cone bean oferece uma imagem tridimensional, sendo a produção desse tipo de imagem através de múltiplos detectores que fracionam a imagem em pequenas fatias de 0,5 a $0,1 \mathrm{~mm}$ o que permite a observação de detalhes de forma funcional ou estática. A imagem é constituída pelo pixel, e a escala Hounsfield é utilizada para traduzir a densidade tecidual sendo o pondo 0 correspondente a densidade da água. Os tecidos mais densos são decodificados com números positivos e nomeados hiperdensos, e os menos densos decodificados com números negativos e nomeados hipodensos. A terceira dimensão corresponde a espessura do corte e é classificada pelo voxel. Ele corresponde a menor unidade de espessura do corte e varia de 0,5 a $20 \mathrm{~mm}$, que é ajustado para ter melhor precisão de acordo com o objetivo (MacDonald, 2017; Nasseh \& Al-Rawi, 2018; Vandenberghe, 2018) .

O objetivo do trabalho é realizar uma revisão de literatura e um relato de caso clínico sobre a presença de múltiplos dentes impactados em um paciente sem síndromes diagnosticadas.

\section{Metodologia}

Esse estudo é uma revisão da literatura e relato de caso realizado por meio de uma abordagem qualitativa com objetivos exploratórios e descritivos (Pereira et al., 2018). As fontes foram obtidas na base de dados eletrônica PubMed. As palavras-chave: "supernumerary multiple", "computed tomography", "dentistry" e "non-syndromic" foram utilizadas durante a coleta da bibliografia juntamente com o operador "AND".

Foram utilizados artigos escritos na língua inglesa, publicados entre 2006 e 2020. O trabalho foi submetido ao Comitê de Ética em Pesquisa sob o número de parecer 4.299.607 e CAAE: 37512920.6.0000.8078.

\section{Relato de Caso}

O paciente do sexo masculino, 28 anos de idade, leucoderma, compareceu a clínica da Faculdade Patos de Minas FPM para avaliação de dentes supranumerários impactados na maxila. Ao procurar tratamento ortodôntico, para alinhar o sorriso e instalação implantes dentários para o espaço edêntulo foi solicitado o exame panorâmico onde observou-se a presença 
de dentes supranumerários impactados. Por motivos de custos e complexidade do caso o paciente foi encaminhado a clínica de cirurgia da Faculdade Patos de Minas - FPM.

Durante a anamnese observou-se ausência de doenças sistêmicas e/ou síndromes, tanto em relação ao paciente quando aos familiares, e a história odontológica não revelou tratamento odontológico prévio, indicando o motivo do diagnóstico tardio.

Durante o exame clínico os sinais observados foram: pequeno diastema e ausência de um dente pré-molar superior esquerdo.

Ao analisar o exame panorâmico observou-se três imagens radiopacas, sugestivas de elemento dentários, sendo duas na região anterior de maxila, próximas a linha média, e outra próxima ao ápice do dente 26. Para um diagnóstico mais apurado, melhor localização das imagens radiopacas e decisão terapêutica foi solicitada uma Tomografia computadorizada.

Na tomografia computadorizada foi observado a imagem sugestiva de dois supranumerários na região média da maxila, sendo um supranumerário, caudal, em posição horizontal, sentido mésio angular, posicionado na região anterior da maxila, com o terço apical da sua raiz posicionado palatinamente em relação ao incisivo lateral superior direito (dente 12). A região do terço médio da raiz próximo a cortical palatina e sua coroa posicionada por palatina em relação ao incisivo central superior direito (dente 11). (Figura 1: A-D).

Figura 1: Tomografia da localização de supranumerários e impactados: (A) Vista tomográfica de dois supranumerários na região média da maxila anterior, sendo um em posição cranial e o outro caudal. (B) Representação tridimensional dos elementos. (C) Corte sagital mediano da representação tridimensional vista esquerda. (D) Corte sagital mediano da representação tridimensional vista direita.

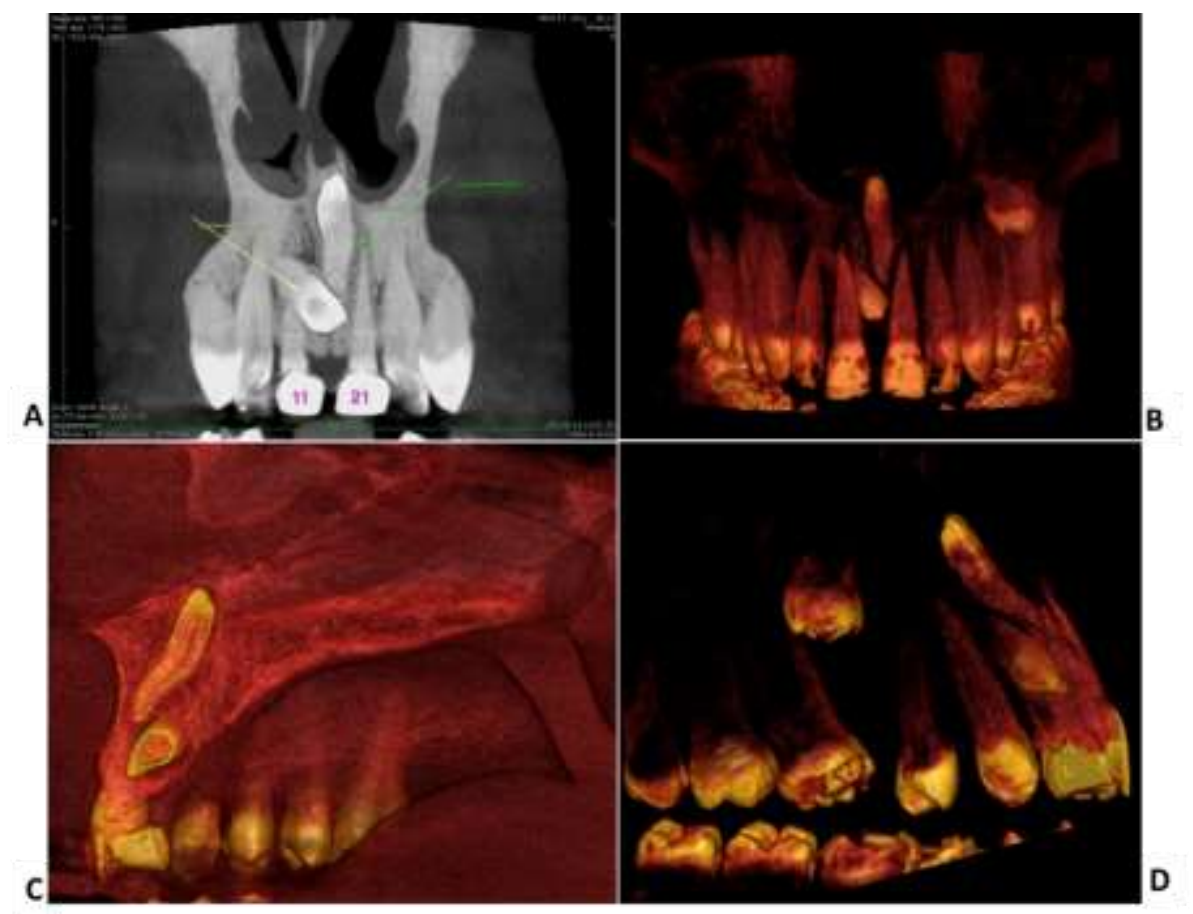

Fonte: Autores (2021).

Notou-se ainda presenta de supranumerário cranial em posição vertical, sentido inverso, também na região anterior da maxila. A coroa estava voltada para o palato, em íntimo contato com o canal incisivo, com a região de terço médio e apical da raiz próximo a coroa do dente supranumerário. Presença de dilaceração radicular. (Figura 2: A-B). 
Figura 2: Cortes para comparação com estruturas próximas: (A) Corte axial com localização dos dentes impactados cranial e caudal. (B) Relação do supranumerário cranial com a fossa nasal.
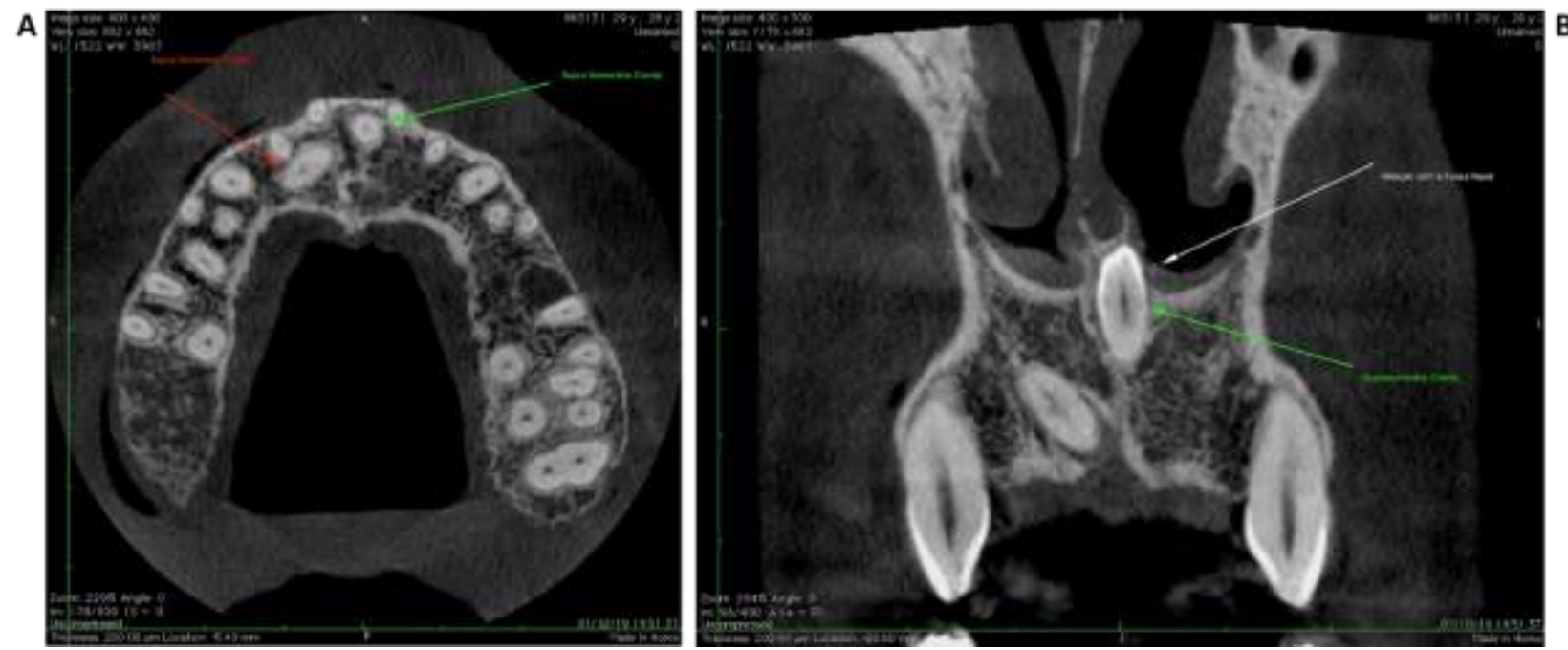

Fonte: Autores (2021).

Dente 25 com rizogênese incompleta em contato com a cortical do seio maxilar esquerdo. (Figura 3: A-B).

Figura 3: Avaliação da localização do dente incluso: (A) Pré-molar incluso e sua relação com o seio maxilar. (B) Pré-molar incluso representação tridimensional.

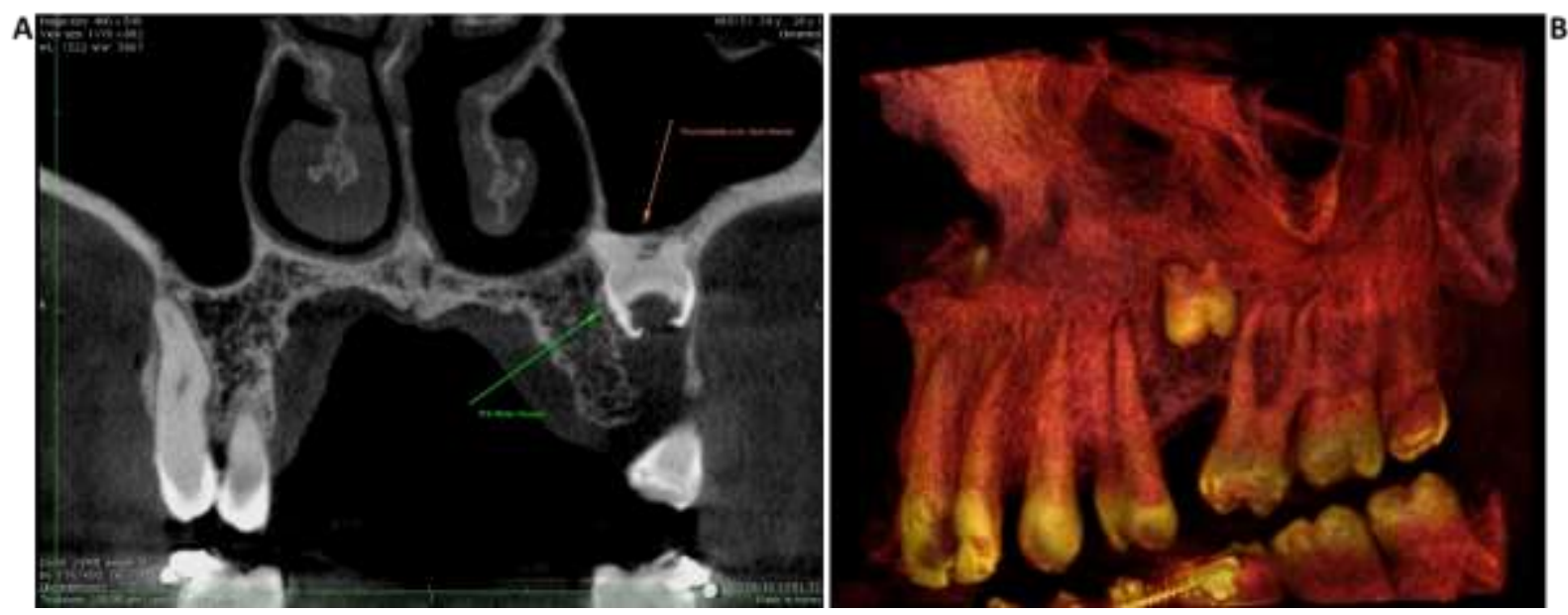

Fonte: Autores (2021).

Diante das imagens obtidas os dentes supranumerários presentes na região anterior de maxila são a provável causa do diastema e o espaço edêntulo corresponde ao dente 25 impactado não erupcionado.

O tratamento recomendado foi a exodontia dos dentes supranumerários e do dente impactado, por não ter um prognóstico favorável para tracionamento ortodôntico devido ao posicionamento e idade do paciente.

\section{Discussão}

As anomalias de número de dentes podem estar envolvidas com o início da formação dos germes dentários devido a modulação inadequada das moléculas de sinalização que controlam esse desenvolvimento, como a proteína morfogenética 
óssea (BMP), o fator de crescimento de fibroblastos (FGF), o fator de necrose tumoral (TNF) e as moléculas do ouriço sônico (Shh) e vias de sinalização (Wnt) (Cobourne \& Sharpe, 2010).

Além da ação das moléculas de sinalização, durante a embriogênese, há na região de diastema brotos dentais que sofreram apoptose através de marcadores inibidores de quinase, a falha nesse processo pode ser, portanto, a responsável pela presença de supranumerários nessa região (Klein et al., 2013).

A presença de múltiplos dentes impactados está diretamente relacionada a presença de alguma síndrome. Apesar disso alguns casos, onde não haviam síndromes relacionadas, foram relatados e os estudos genéticos fizeram-se necessários (Bae et al., 2017). Bae, et al., 2017, avaliou geneticamente, através da saliva, nove indivíduos de uma família com pré-molares supranumerários em duas gerações sucessivas. Foram analisados cem prováveis genes envolvidos na regulação do desenvolvimento dentário. Dos nove membros da família avaliados, quatro foram afetados, sendo todos homens, o que leva a afirmação de supranumerários herdados de forma autossômica dominante.

De acordo com a revisão de Anthonappa, et al, 2013 e Tanwar, et al., 2017 as prováveis causas de dentes supranumerários são atavismo, dicotomia, hiperatividade da lâmina dentária, hereditariedade, teoria da zona de progresso e etiologia unificada (Anthonappa et al., 2013; Tanwar et al., 2017). A teoria da lâmina dentária é a mais aceita até hoje. Acredita-se que esse excesso de atividade da lâmina está relacionado ao desenvolvimento anormal do embrião devido a fatores genéticos e uma estimulação através de fatores ambientais, ou seja, multifatorial (Lu et al., 2017).

Para completar o diagnóstico, além de toda história médica e odontológica, são necessários os exames complementares de imagem. Nasseh e Al Rawi, 2018 mostraram em seu trabalho as várias aplicações da tomografia computadorizada na área odontológica, entre elas, localização das características anatômicas e anormalidades de desenvolvimento, sem ampliação e sobreposições e contraste bastante satisfatório, auxiliando o correto diagnóstico e planejamento do tratamento.

O caso é relevante para a literatura visto que existem poucos casos de dentes supranumerários não relacionados a síndromes, e ainda menos quando se trata de múltiplos dentes supranumerários, levando a crer que a correlação genética deve ser cada vez mais estudada.

\section{Considerações Finais}

Os dentes supranumerários em pacientes não sindrômicos são raros e podem estar relacionados a alterações na modulação das moléculas de sinalização que controlam o desenvolvimento dental, ou na falha dos marcadores inibidores de quinase, responsáveis por apoptose dos brotos dentais, principalmente na região de diastema.

Diante de um quadro com múltiplos dentes supranumerários é importante a pesquisa sobre outras possíveis características clínicas, imaginológicas e sistêmicas compatíveis com síndromes, principalmente, craniofaciais.

Uma avaliação imaginológica detalhada, preferencialmente com imagens tomográficas permitem um diagnóstico preciso e uma intervenção cirúrgica precoce minimizando as repercussões clínicas associadas a retenção tardia desses dentes.

\section{Agradecimentos}

Financiamento: Este estudo também foi parcialmente financiado pela CAPES - Código Financeiro 001. Agradecemos também o apoio do CNPq (Conselho de Desenvolvimento Científico e Tecnológico - Brasil) - Código Financeiro 307808 / 2018-1 


\section{Referências}

Açıkgöz, A., Açıkgöz, G., Tunga, U., \& Otan, F. (2006). Characteristics and prevalence of non-syndrome multiple supernumerary teeth: A retrospective study. Dentomaxillofacial Radiology, 35(3), 185-190. https://doi.org/10.1259/dmfr/21956432

Al-Tamimi, B., Abela, S., Jeremiah, H. G., \& Evans, R. D. (2017). Supernumeraries in Nicolaides-Baraitser Syndrome. International Journal of Paediatric Dentistry, 27(6), 583-587. https://doi.org/10.1111/ipd.12309

Anthonappa, R. P., King, N. M., \& Rabie, A. B. M. (2013). Aetiology of supernumerary teeth: A literature review. European Archives of Paediatric Dentistry, 14(5), 279-288. https://doi.org/10.1007/s40368-013-0082-Z

Bae, D. H., Lee, J. H., Song, J. S., Jung, H.-S., Choi, H. J., \& Kim, J. H. (2017). Genetic analysis of non-syndromic familial multiple supernumerary premolars. Acta Odontologica Scandinavica, 75(5), 350-354. https://doi.org/10.1080/00016357.2017.1312515

Belmehdi, A., Bahbah, S., El Harti, K., \& El Wady, W. (2018). Non syndromic supernumerary teeth: Management of two clinical cases. Pan African Medical Journal, 29. https://doi.org/10.11604/pamj.2018.29.163.14427

Boeddinghaus, R., \& Whyte, A. (2008). Current concepts in maxillofacial imaging. European Journal of Radiology, 66(3), 396-418. https://doi.org/10.1016/j.ejrad.2007.11.019

Chen, K.-C., Huang, J.-S., Chen, M.-Y., Cheng, K.-H., Wong, T.-Y., \& Huang, T.-T. (2019). Unusual Supernumerary Teeth and Treatment Outcomes Analyzed for Developing Improved Diagnosis and Management Plans. Journal of Oral and Maxillofacial Surgery, 77(5), 920-931. https://doi.org/10.1016/j.joms.2018.12.014

Cobourne, M. T., \& Sharpe, P. T. (2010). Making up the numbers: The molecular control of mammalian dental formula. Seminars in Cell \& Developmental Biology, 21(3), 314-324. https://doi.org/10.1016/j.semcdb.2010.01.007

Dodson, T. B. (2012). The Management of the Asymptomatic, Disease-Free Wisdom Tooth: Removal Versus Retention. Atlas of the Oral and Maxillofacial Surgery Clinics, 20(2), 169-176. https://doi.org/10.1016/j.cxom.2012.06.005

Khalaf, K., Al Shehadat, S., \& Murray, C. A. (2018). A Review of Supernumerary Teeth in the Premolar Region. International Journal of Dentistry, 2018, 16. https://doi.org/10.1155/2018/6289047

Klein, O. D., Oberoi, S., Huysseune, A., Hovorakova, M., Peterka, M., \& Peterkova, R. (2013). Developmental disorders of the dentition: An update: American Journal Of Medical Genetics Part C (Seminars In Medical Genetics). American Journal of Medical Genetics Part C: Seminars in Medical Genetics, 163(4), 318-332. https://doi.org/10.1002/ajmg.c.31382

Lu, X., Yu, F., Liu, J., Cai, W., Zhao, Y., Zhao, S., \& Liu, S. (2017). The epidemiology of supernumerary teeth and the associated molecular mechanism. Organogenesis, 13(3), 71-82. https://doi.org/10.1080/15476278.2017.1332554

MacDonald, D. (2017). Cone-beam computed tomography and the dentist. Journal of Investigative and Clinical Dentistry, 8(1), e12178. https://doi.org/10.1111/jicd.12178

Main genetic entities associated with supernumerary teeth. (2018). Archivos Argentinos de Pediatria, 116(6). https://doi.org/10.5546/aap.2018.eng.437

McCoy, J. M. (2012). Complications of Retention: Pathology Associated with Retained Third Molars. Atlas of the Oral and Maxillofacial Surgery Clinics, 20(2), 177-195. https://doi.org/10.1016/j.cxom.2012.06.002

Meara, D. J. (2012). Evaluation of Third Molars: Clinical Examination and Imaging Techniques. Atlas of the Oral and Maxillofacial Surgery Clinics, 20(2), 163-168. https://doi.org/10.1016/j.cxom.2012.07.001

Nasseh, I., \& Al-Rawi, W. (2018). Cone Beam Computed Tomography. Dental Clinics of North America, 62(3), 361-391. https://doi.org/10.1016/j.cden.2018.03.002

Pereira, A. S., Shitsuka, D. M., Parreira, F. J., \& Shitsuka, R. (2018). Metodologia do trabalho científico. [e-Book]. Santa Maria. Ed. UAB/NTE/UFSM. https://repositorio.ufsm.br/bitstream/handle/1/15824/Lic_Computacao_Metodologia-Pesquisa-Cientifica.pdf?sequence=1

Pico, C., do Vale, F., Caramelo, F., Corte-Real, A., \& Pereira, S. (2017). Comparative analysis of impacted upper canines:Panoramic radiograph Vs Cone Beam Computed Tomography. Journal of Clinical and Experimental Dentistry, e1176-e1182. https://doi.org/10.4317/jced.53652

Tamimi, D., \& ElSaid, K. (2009). Cone Beam Computed Tomography in the Assessment of Dental Impactions. Seminars in Orthodontics, 15(1), 57-62. https://doi.org/10.1053/j.sodo.2008.09.007

Tanwar, R., Jaitly, V., Sharma, A., Heralgi, R., Ghangas, M., \& Bhagat, A. (2017). Non-syndromic multiple supernumerary premolars: Clinicoradiographic report of five cases. Journal of Dental Research, Dental Clinics, Dental Prospects, 11(1), 48-52. https://doi.org/10.15171/joddd.2017.009

Vandenberghe, B. (2018). The digital patient - Imaging science in dentistry. Journal of Dentistry, 74, S21-S26. https://doi.org/10.1016/j.jdent.2018.04.019 